\title{
Assessment of gas flow characteristics and reaction kinetics of chemical vapour deposition reactors for the production of nano-hybrid fibres
}

\begin{abstract}
Chemical vapour deposition (CVD) had been used to produce carbon nanotube (CNT) coated carbon fibre, referred to in this work as nano-hybrid fibres. In order to understand the gas flow behaviour inside the CVD reactor, computational fluid dynamics (CFD) has been utilised to simulate the process in a horizontal CVD reactor together with a vertical CVD reactor for comparison. For simplicity purposes, the CNT synthesis was represented by ferrocene decomposition into iron, which acts as the catalyst for CNT formation. The gas flow behaviour inside the horizontal reactor was asymmetrical in nature and seen to be dominated by recirculation effects compared with the vertical reactor, which was symmetrical and generally lacking in circulation. The impact of the gas flow characteristics on the reaction rates of the ferrocene decomposition and mass fraction of chemical species was also investigated. The kinetic rate of reaction in the horizontal reactor was found to be 2-3 orders of magnitude larger in the horizontal reactor compared with the vertical reactor. The results provide valuable insight into the best approach for producing nano-hybrid fibres in a one-step continuous process.
\end{abstract}

Keyword: Chemical vapour deposition; Gas flow; Reaction kinetics; Computational fluid dynamics 